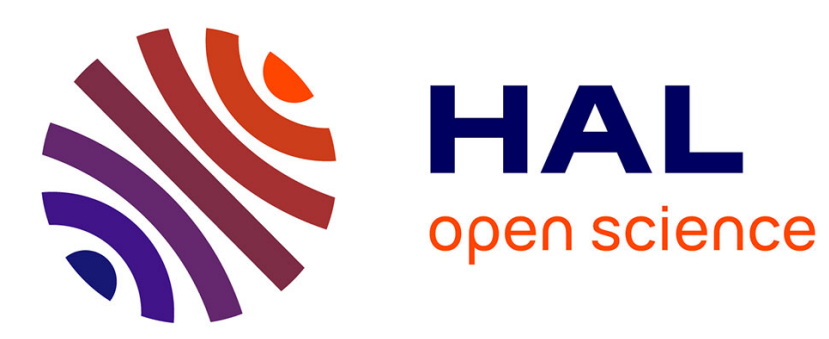

\title{
Electron states and recombination velocities at semiconductor surfaces and interfaces
}

M. Lannoo

\section{To cite this version:}

M. Lannoo. Electron states and recombination velocities at semiconductor surfaces and interfaces. Revue de Physique Appliquée, 1987, 22 (8), pp.789-795. 10.1051/rphysap:01987002208078900 . jpa00245612

\section{HAL Id: jpa-00245612 https://hal.science/jpa-00245612}

Submitted on 1 Jan 1987

HAL is a multi-disciplinary open access archive for the deposit and dissemination of scientific research documents, whether they are published or not. The documents may come from teaching and research institutions in France or abroad, or from public or private research centers.
L'archive ouverte pluridisciplinaire HAL, est destinée au dépôt et à la diffusion de documents scientifiques de niveau recherche, publiés ou non, émanant des établissements d'enseignement et de recherche français ou étrangers, des laboratoires publics ou privés. 


\title{
Electron states and recombination velocities at semiconductor surfaces and interfaces
}

\author{
M. Lannoo \\ Laboratoire des Surfaces et Interfaces (*) Institut Supérieur d'Electronique du Nord, 41, boulevard Vauban, \\ 59046 Lille Cedex, France
}

(Reçu le 2 février 1987, accepté le 16 avril 1987)

\begin{abstract}
Résumé. - Le concept d'état associé aux liaisons pendantes est passé en revue. Il est montré que la hauteur de barrière de Schottky peut être corrélée à l'énergie propre moyenne des liaisons pendantes de surface. La même propriété s'avère vraie pour les discontinuités de bandes aux hétérojonctions entre semiconducteurs. Le modèle explique la relation trouvée par Tersoff entre ces deux quantités. La deuxième partie est consacrée à la détermination théorique des vitesses de recombinaison de surface. Il est montré que le modèle de StevensonKeyes est capable de donner des vitesses de recombinaison de l'ordre de $10^{5}$ à $10^{7} \mathrm{~cm} / \mathrm{s}$. Des travaux récents sur InP sont discutés. Finalement une expression semi-classique des sections de capture multiphonons est utilisée, apportant des informations supplémentaires sur les états de surface qui dominent la recombinaison.
\end{abstract}

\begin{abstract}
The concept of dangling bond states is reviewed. It is shown that the Schottky barrier height can be correlated with the average self energy of surface dangling bonds. The same property holds true for band offsets at semiconductor heterojunctions. The model explains the relation found by Tersoff between these two quantities. A second part is devoted to the theoretical determination of surface recombination velocities. The Stevenson-Keyes model is shown to be able to provide recombination velocities of order $10^{5}$ to $10^{7} \mathrm{~cm} / \mathrm{s}$. Recent work on InP is discussed. Finally a semiclassical expression of the multiphonon capture cross sections is used, providing more information on the surface states which dominate recombination.
\end{abstract}

\section{Introduction.}

Semiconductor device performance is often governed by the existence of surface or interface states. Such states can be due to the presence of defects, for instance trivalent silicon at the $\mathrm{Si}_{-} \mathrm{SiO}_{2}$ interface or excess arsenic at the GaAs-oxyde interface. Fermi level pinning at Schottky barriers is also due to interface states which are either metal induced gap states [1] or defect induced states [2]. Even the side surfaces of field effect transistors exhibit defect states which play an important role in device operation.

One aim of this paper is first to present evidence that most of the experimental results can be connected together through the concept of dangling bonds. Such dangling bonds naturally occur at free surfaces and for trivalent silicon at the $\mathrm{Si}-\mathrm{SiO}_{2}$ interface. However we show that they must be important as resonant states at Schottky barriers and

(*) Unité Associée au C.N.R.S. $\mathrm{N}^{\circ} 253$ that they implicitely govern band offsets at semiconductor heterojunctions. They thus provide a simple physical basis to understand Tersoff's model relating Schottky barrier heights and band offsets. They are also important for many kinds of defects.

The second part will be devoted to a discussion of the surface recombination velocity $S$. We reexamine the Stevenson-Keyes formula [3, 4] both in the low and high injection regimes. We show that it can provide reasonable orders of magnitude for $S$ $\left(\sim 10^{5}\right.$ to $\left.10^{7} \mathrm{~cm} / \mathrm{s}\right)$ in a variety of situations. We then make an estimate of the multiphonon capture cross-sections using a semiclassical theory valid in the large temperature limit. We apply it to recent work on InP and show that it can serve as a test of the coherence between spectroscopic and electric informations.

\section{The nature of dangling bond states.}

We only consider here zinc-blende semiconductors which are characterized by the existence of four 
covalent (and eventually partly ionic) bonds. Any time such bonds are broken (i.e. at vacancies, surfaces, etc.) there remain undercoordinated atoms with dangling bonds. There is ample experimental evidence that these dangling bonds induce gap states. The best known case is certainly the vacancy in silicon for which several dangling bond levels corresponding to different charge states have been observed [5]. However by far the simplest system corresponds to the isolated dangling bond characteristic of the trivalent silicon atoms which exist in a $\mathrm{Si}$ or at the $\mathrm{Si}_{-} \mathrm{SiO}_{2}$ interface. Such a dangling bond is characterized by one gap level $[6,7]$ which can be filled by zero, one or two electrons, leading respectively to the $\mathrm{D}^{+}, \mathrm{D}^{0}$ and $\mathrm{D}^{-}$charge states. Experimental values of the energy levels correspond to ionization energies which obviously depend on charge state.

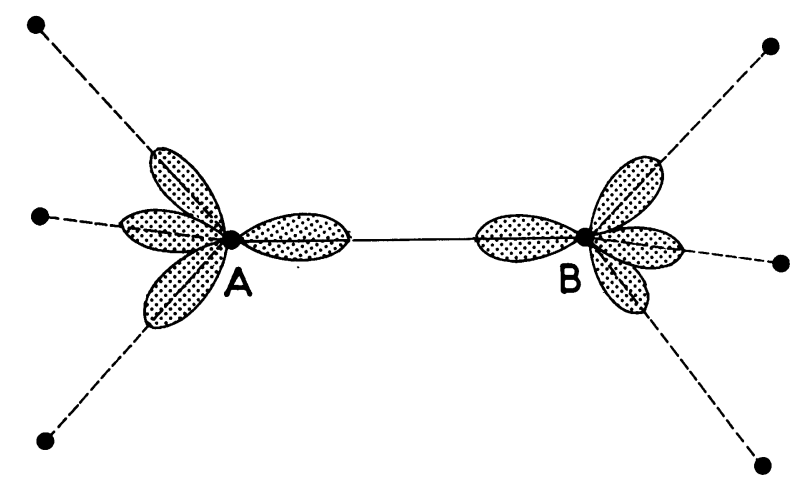

Fig. 1. - $\mathrm{sp}^{3}$ hybrid orbitals involved in a covalent bond.

The simplest understanding of the origin of dangling bond states comes from the tight binding approximation. Here the wave function of the valence electrons is expanded in a minimal free atom basis set consisting of one « $s$ » and three $\langle p$ » wave functions per atom. To classify the interactions by order of importance it is best to perform a basis change and build four equivalent $\mathrm{sp}^{3}$ combinations on every atom, each of them strongly pointing towards a nearest neighbour (see Fig. 1). The dominant interactions will thus connect pairs of $\mathrm{sp}^{3}$ hybrids involved in the same bond. The energy level scheme of such pairs is analogous to the one of diatomic molecules and consists in one bonding state filled with two electrons and one empty antibonding state. Interactions between bonds broaden the bonding states into the valence band and the antibonding states into the conduction band (Fig. 2). In such a scheme the dangling bonds correspond to a $\mathrm{sp}^{3}$ hybrid that has no partner. To lowest order its energy will remain the $\mathrm{sp}^{3}$ atomic energy. Further interactions act to shift it slightly so that it becomes a gap state as shown in figure 2 .

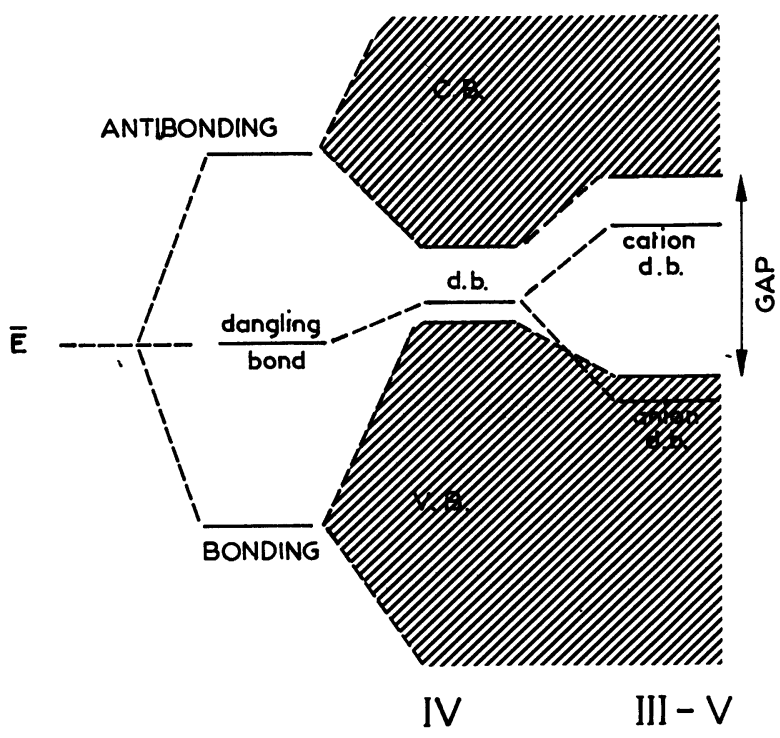

Fig. 2. - Formation of the valence and conduction bands in zinc-blende semiconductors from the bonding and antibonding levels. Also shown is the dangling bond energy which, in the III-V, splits into two levels : one for cations, one for anions.

This simple picture allows to understand easily the behaviour of surface states. For instance the unreconstructed $\mathrm{Si}(111)$ surface corresponds to an array of parallel dangling bonds. Their interaction broadens the isolated dangling bond state into a half filled band (since the neutral surface corresponds to one electron per dangling bond). Under a $2 \times 1$ reconstruction this band splits into a filled and an empty subband (Fig. 3a). The case of the (110) face of III-V compounds is slightly different. There is now one anion and one cation dangling bond the former one being at lower energy. The situation for the unrelaxed surface is shown in figure $3 \mathrm{~b}$. However relaxation acts to shift these bands in such a way that the gap is emptied as shown in figure 3c. The (100) faces are a little more complex involving some amount of dimerization.

\section{Dangling bonds, Schottky barrier heights and band offsets.}

It has been shown for some time that Schottky barriers on most III-V semiconductors are characterized by a pinning of the Fermi level (see Ref. [8] for a review) which is practically independent of the nature of the metal. Such pinning already occurs at a fraction $(\sim 0.2)$ of a monolayer of deposited metal. These facts have led Spicer to propose his defect model [2] based on the hypothesis that the Fermi level is pinned on gap states, induced by semiconductor surface defects, whose position might remain independent of the nature of the metal. This model has been analysed theoretically by Sankey et al. [9] 

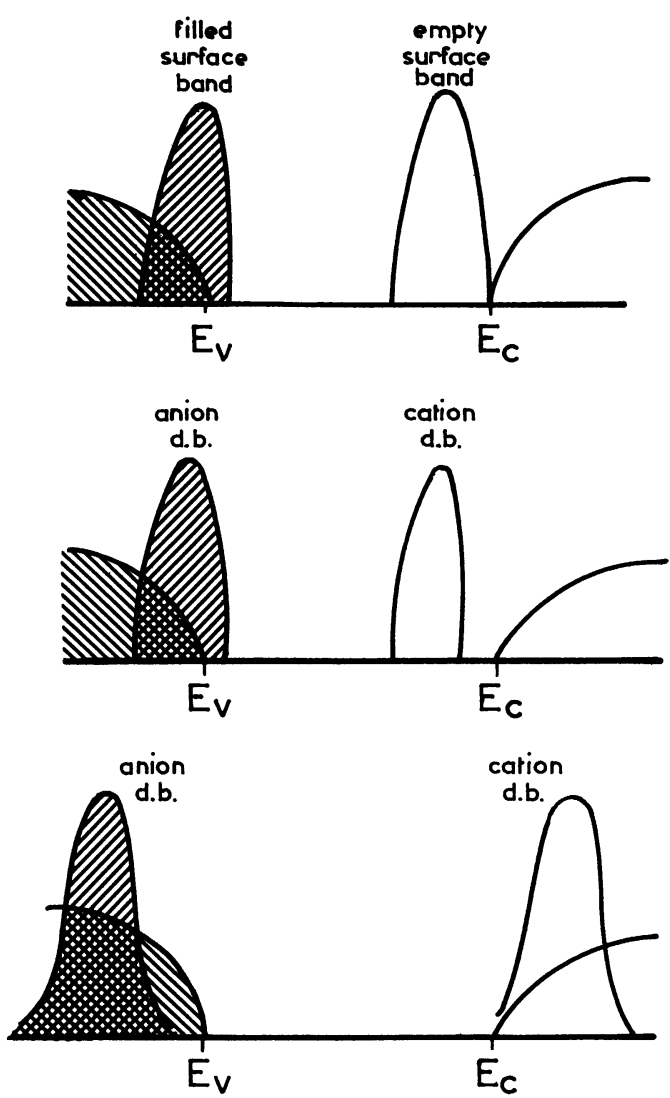

b)

c)

Fig. 3. - Schematic representation of the dangling bond surface bands for : a) $\mathrm{Si}(111) 2 \times 1$, b) $\mathrm{GaAs}(110)$ before reconstruction, c) $\mathrm{GaAs}(110)$ after reconstruction.

who indeed obtained, from a tight binding calculation, a striking correlation between the Fermi level position $E_{\mathrm{FS}}$ at the surface and gap states corresponding to surface cation antisites.

On the other hand Tersoff [10] has pointed out that there is a relation between $E_{\mathrm{FS}}$ and the band offset at semiconductor heterojunctions. Tersoff's theory of Schottky barrier heights is based on the existence of metal induced gap states $[1,8]$, i.e. a continuum of states that overlaps the semiconductor gap and whose wave functions decay exponentially into the semiconductor. For the semiconductor it uses the concept of a charge neutrality level $E_{\mathrm{B}}$ (introduced by Tejedor et al. [11]) which plays a role analogous to the Fermi level $E_{\mathrm{F}}$ in a metal. If one accepts this view the Schottky barrier height is then determined by aligning $E_{\mathrm{F}}$ and $E_{\mathrm{B}}$ characteristic of each semiconductor.

A direct check of this theory can be made by considering the interface between $\mathrm{Au}$ and many semiconductors. If one uses the observed barrier heights as experimental values of $E_{\mathrm{B}}$, then one gets good predictions for the band offsets. Tersoff has also proposed a more or less ad hoc theoretical rule to determine $E_{\mathrm{B}}[10]$ from the bulk semiconductor band structure. Although this rule works remarkably well its physical origin is not clear. In particular heterojunctions do not necessarily involve interface states and $E_{\mathrm{B}}$ should be related in some way to surface dangling bond states. It is this relation which is examined in the following where $E_{\mathrm{B}}$ will be identified with the average self energy of dangling bonds.

Before discussing this point further it is useful to have a look at the present state of the theory concerning the predictions of band offsets. A recent review of this matter and of Schottky barriers is given in reference [8]. In practice available theories of band offsets are not capable of predicting them with the desired accuracy of $0.1 \mathrm{eV}$. Even the experimental values are not yet completely reliable [12]. At present the most successful predictions have been obtained from simple semiempirical theories. However there have also been systematic local density [13] as well as tight binding calculations $[14,15]$. Typical results are given in table $\mathbf{I}$ showing the kind of agreement that can be obtained.

Table I. - Theoretical and experimental values of valence band offsets (in $\mathrm{eV})$ for different heterojunctions.

\begin{tabular}{|l|c|c|c|c|c|c|}
\hline \multicolumn{1}{|c|}{ Heterojunction } & (a) & (b) & (c) & (d) & (e) & Experiment \\
\hline AlAs-GaAs & 0.37 & $0.49-0.63$ & 0.55 & 0.48 & 0.46 & $0.19[16] 0.38[17]$ \\
& & & & & & $0.45-0.56[18,19]$ \\
InAs-GaSb & 0.38 & 0.27 & 0.43 & 0.23 & 0.12 & $0.46[20]$ \\
AsGa-Ge & 0.63 & 0.62 & 0.32 & 0.42 & 0.56 & $0.35[21] 0.56[22]$ \\
GaP-Si & 0.61 & 0.48 & 0.45 & 0.35 & 0.69 & $0.80[21]$ \\
AlAs-Ge & 1.05 & 0.89 & 0.87 & 0.91 & 1.02 & $0.95[21]$ \\
ZnSe-Ge & 2.17 & 1.62 & 1.52 & & & $1.40[23] 1.52[24]$ \\
ZnSe-GaAs & 1.58 & 1.01 & 1.20 & & & $0.96[24]$ \\
\hline
\end{tabular}
(a) Theory, Local density [13].
(b) Theory, Tight binding local neutrality [14].
(c) Theory, Tersoff's model [10].
(d) This work with the parameters of reference [27].
(e) This work with the parameters of reference [28]. 
Let us now come back to the relation between $E_{\mathrm{B}}$ and the dangling bond states. For this we first examine the interface between a metal and a covalent semiconductor. As we have seen in section 2 the semiconductor surface is characterized by the existence of localized dangling bond states. It is their coupling with the metal states that will dominate the electronic properties of the interface. To simplify let us consider the coupling of each dangling bond separately. The situation is pictured schematically in figure $4 \mathrm{a}$ where one can see that the dangling bond state becomes a resonant state, i.e. is shifted and broadened by its interaction with the continuum of metallic states. The broadened density of states contains one state per dangling bond. The metal Fermi level $E_{\mathrm{F}}$ must then align with the barycentre $E_{\mathrm{B}}$ of this density of states. For reasonably symmetrical coupling the shift of the resonant state with respect to the free surface energy is small so that $E_{\mathrm{B}} \sim E_{\mathrm{db}}$, the free surface dangling bond energy. The conclusion is then that the role of Tersoff's

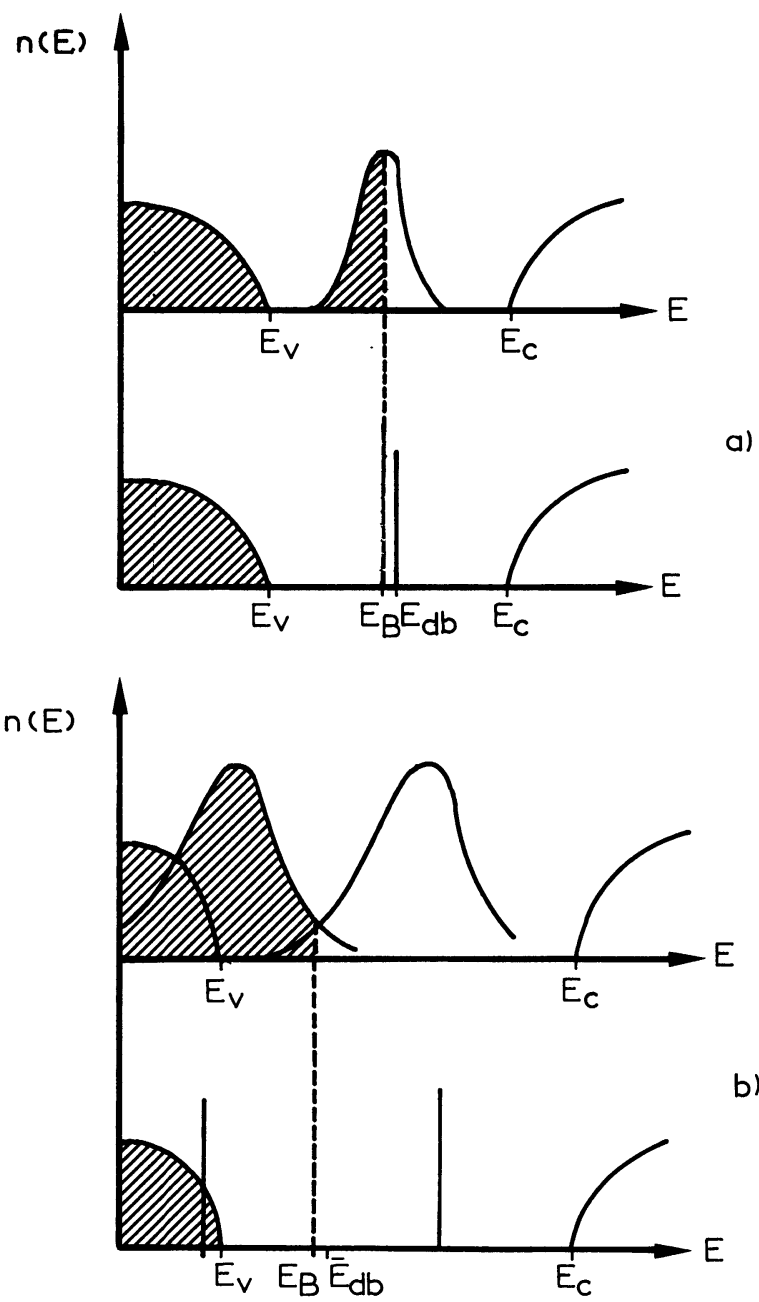

Fig. 4. - Broadening of the dangling bond stater into a resonant state : a) for covalent semiconductors, b) for a non-polar face of III-V semiconductors. neutrality level is played by the dangling bond energy $E_{\mathrm{db}}$.

This reasoning can be extended to the non-polar surfaces of compounds. In such cases there are one anion and one cation dangling bond levels which are broadened as shown in figure $4 \mathrm{~b}$. There are two states and two electrons per pair of dangling bonds. For reasonably symmetrical situations $E_{\mathrm{F}}$ must now align with $\bar{E}_{\mathrm{db}}=\frac{E_{\mathrm{dbA}}+E_{\mathrm{dbC}}}{2}$ the average dangling bond energy.

A more mathematical derivation of the identification $E_{\mathrm{B}}=\bar{E}_{\mathrm{db}}$ is given in reference [25]. To substantiate this identification we have also performed a tight binding calculation of the position $\bar{E}_{\mathrm{db}}$ for covalent and III-V semiconductors by a selfconsistent tight binding technique analogous to that used for the vacancy in Si $[14,26]$ and at heterojunctions. The results are compared in figure 5 , for two sets of parameters [27, 28], to the experimental position of the surface Fermi level for Au on different semiconductors. The agreement is impressive and the trend is clearly reproduced for both sets of parameters.

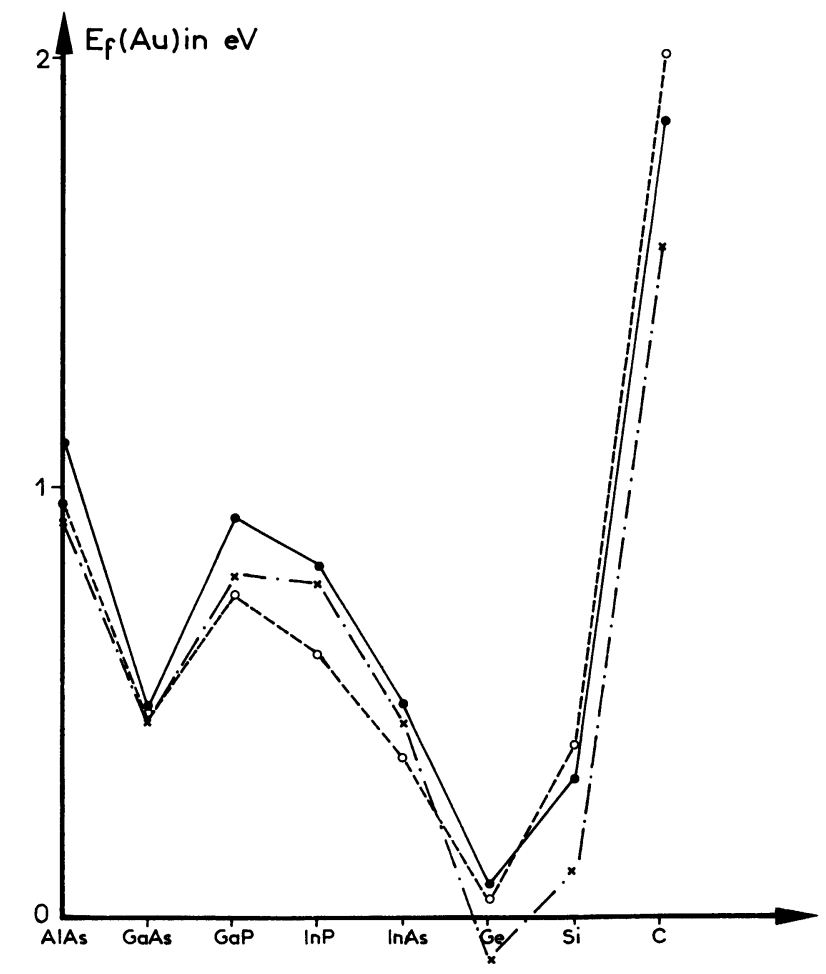

Fig. 5. - Surface Fermy energy for $\mathrm{Au}$ on different semiconductors. Comparison between the experimental value (-) and the average dangling bond energy obtained with the parameters of reference [27] (---------) or reference [28] (-.-.--.).

It remains to show why band offsets at heterojunctions should be related to $\bar{E}_{\mathrm{db}}$. For lattice matched 
systems dangling bond states will couple by pairs across the interface to form bonding and antibonding states. For one type of dangling bond charge transfer across the interface is directly imposed by the difference in $E_{\mathrm{db}}$ on each side. However in such covalent semiconductors screening is very efficient and this charge transfer is practically reduced to zero, which corresponds to an alignment of $\bar{E}_{\mathrm{db}}$ on each side. The argument can be extended to two types of dangling bonds [25] showing that $\bar{E}_{\mathrm{db}}$ plays the role of a surface Fermi level, exactly as was postulated by Tersoff. The theoretical offsets obtained in this way are compared to experiment in table I.

\section{Recombination velocities.}

As pointed out by Aspnes [29] there is a difficulty in understanding the observed values of the recombination velocity $\left(\sim 10^{5}\right.$ to $\left.10^{7} \mathrm{~cm} / \mathrm{s}\right)$ when using the Stevenson-Keyes formula [3, 4]. We shall then reexamine this problem in the light of recent work [30] and with the help of theoretical expressions for the capture cross-sections. The Stevenson-Keyes expression is valid when the following conditions are realized : i) steady state, ii) constant quasi-Fermi levels across the space charge region and iii) injected carrier densities much smaller than bulk doping. Then, the surface recombination velocity $S$ can be written [4] :

$$
S=\frac{v \sigma N_{\mathrm{S}}\left(n_{\mathrm{b}}^{0}+p_{\mathrm{b}}^{0}\right)}{2 n_{i}\left\{\cosh \left(\frac{E_{\mathrm{T}}-E_{i}}{k T}-u_{0}\right)+\exp \left(\frac{F_{\mathrm{n}}-F_{\mathrm{p}}}{2 k T}\right) \cosh \left(\frac{F-E_{i}}{k T}-u_{0}\right)\right\}}
$$

In this expression $N_{\mathrm{S}}$ is the number of recombination centers per unit surface, of electrical level $E_{\mathrm{T}}$, $n_{\mathrm{b}}^{0}$ and $p_{\mathrm{b}}^{0}$ are the equilibrium bulk carrier densities, $n_{i}$ and $E_{i}$ are the intrinsic concentration and surface Fermi level, $F_{\mathrm{n}}$ and $F_{\mathrm{p}}$ are the quasi Fermi levels and $\bar{F}$ their average. The term $v \sigma$ represents the quantity $\sqrt{K_{\mathrm{n}} K_{\mathrm{p}}}$, where $K_{\mathrm{n}}$ and $K_{\mathrm{p}}$ are the capture probabilities defined in [4]. These are usually rewritten as products of thermal velocities $\left(v_{n}, v_{p}\right)$ times capture cross-sections $\left(\sigma_{n}, \sigma_{\mathrm{p}}\right)$. Thus $v$ and $\sigma$ are geometrical averages of these quantities. Finally, as in reference [4] we define $u_{0}$ by

$$
u_{0}=l_{n} \sqrt{\frac{K_{\mathrm{p}}}{K_{\mathrm{n}}}}
$$

which reflects the asymmetry in capture probabilities.

A first estimation can be made in a simple situation where the injection level is low $\left(F_{\mathrm{n}}=F_{\mathrm{p}}\right)$, the Fermi level is pined on $E_{\mathrm{T}}$ and $u_{0}=0$. With reasonable values appropriate to $\mathrm{GaAs}$ or $\mathrm{InP}$ $\left(v=10^{7} \mathrm{~cm} / \mathrm{s}, \quad N_{\mathrm{S}} \sim 10^{13} \mathrm{~cm}^{-2}, \quad n_{\mathrm{b}}^{0}=10^{17} \mathrm{~cm}^{-3}\right.$, $n_{i}=10^{7} \mathrm{~cm}^{-3}$ ) one gets

$$
S=\frac{2.5\left(\frac{\sigma}{10^{-15} \mathrm{~cm}^{2}}\right)}{\cosh \left(\frac{E_{\mathrm{T}}-E_{i}}{k T}-u_{0}\right)} 10^{14} \mathrm{~cm} / \mathrm{s} .
$$

Under conditions where pinning is at midgap $E_{\mathrm{T}}=E_{i}, u_{0}=0$ and $\sigma \sim 10^{-15} \mathrm{~cm}^{2}$ then $S$ is of order $10^{14} \mathrm{~cm} / \mathrm{s}$ i.e. about seven to nine order of magnitudes larger than observed values. This huge discrepancy can be remedied by reducing $\sigma$ to $10^{-22}$, $10^{-24} \mathrm{~cm}^{2}$ (considered as unphysically small by sev- eral authors [29, 30]) or by increasing the denominator

$$
\left(\left|\frac{E_{\mathrm{T}}-E_{\mathrm{I}}}{k T}-u_{0}\right| \sim 16 \text { to } 21\right),
$$

or by any intermediate combination of the two.

However recent work on InP [30] has pointed out the importance of taking into account the influence of the injection level in expression (1). In their situation where $F_{\mathrm{n}}-F_{\mathrm{p}}=1 \mathrm{eV}, \quad \bar{F}-E_{i}=$ $-0.2 \mathrm{eV}, \quad n_{\mathrm{b}}^{0} \sim 3 \times 10^{17} \mathrm{~cm}^{-3}, \quad n_{i}=2 \times 10^{7} \mathrm{~cm}^{-3}$, $N_{\mathrm{S}}=10^{14} \mathrm{~cm}^{-2}$ it is reasonable to assume that, in (1), the denominator is dominated by the second term. One then gets

$$
S=\frac{1.5\left(\frac{\sigma}{10^{-15} \mathrm{~cm}^{2}}\right)}{\cosh \left(\frac{\bar{F}-E_{i}}{k T}-u_{0}\right)} 10^{7} \mathrm{~cm} / \mathrm{s} .
$$

If, with the authors [30], one assumes that $u_{0}=$ 0 , then one gets a correct order of magnitude $S \sim 10^{5} \mathrm{~cm} / \mathrm{s}$ with $\sigma=10^{-15} \mathrm{~cm}^{2}$. However this answer is not unique and it is important to have some feeling about the value which should be expected for $\sigma$.

For this we make use of the theory of capture with multiphonon emission that has been developed for point defects. In this case non-radiative transitions are induced by the electron-lattice coupling and, at high enough temperatures, one can use a semiclassical treatment which leads to

$$
\sigma_{\alpha}=\sigma_{\alpha, 0} \exp -\frac{E_{\mathrm{B} \alpha}}{k T}
$$


where $\alpha=\mathrm{n}$ or $\mathrm{p}$ and $\sigma_{\alpha, 0} \sim 10^{-13} \mathrm{~cm}^{2}[31,32]$. If one neglects the smooth temperature dependence of the prefactor, then $E_{\mathrm{Bn}}$ and $E_{\mathrm{Bp}}$ represent simply the classical barriers for capture pictured on the configuration coordinate diagram in figure 6 . This diagram represents the relevant total electronic energies of the system as a function of one normalized lattice coordinate $Q$ assumed to dominate the electronlattice coupling. To fix the ideas we consider a single donor state and draw three curves : $E_{\mathrm{v}}(Q)$ when the defect is in its positive charge state, $E_{\mathrm{c}}(Q)$ after excitation of an electron-hole pair through the gap, and $E_{0}(Q)$ when the defect has captured an electron and there remains a free hole in the valence band. The simplest treatment assumes that the three curves are parabola with the same curvature, $E_{0}(Q)$ being shifted by an amount $Q_{0}$. The parameters defining the problem are $\varepsilon_{0}$ and the FranckCondon shift $d_{\mathrm{FC}}$ defined in figure 6 . It is easy to show that

$$
E_{\mathrm{Bp}}=\frac{\left(\frac{E_{\mathrm{G}}}{2} \pm \varepsilon_{0}\right)^{2}}{4 d_{\mathrm{FC}}}
$$

From this one directly deduces

$$
\sigma=\sigma_{0} \exp -\left(\frac{\varepsilon_{0}^{2}+\left(\frac{E_{\mathrm{G}}}{2}\right)^{2}}{4 d_{\mathrm{FC}} k T}\right)
$$

and

$$
u_{0}=-\frac{E_{\mathrm{G}}}{4 d_{\mathrm{FC}}} \frac{\varepsilon_{0}}{k T} .
$$

A first remark is that, since $\sigma_{0} \sim 10^{-13} \mathrm{~cm}^{2}$, it is hard to get $\sigma$ of order $10^{-15} \mathrm{~cm}^{2}$ since, even in the most favorable case where $\varepsilon_{0} \sim 0$ this would imply that $d_{\mathrm{FC}} \sim 1 \mathrm{eV}$, which is an unrealistically large value. The only way to get some reasonable answer from (4) would be to assume that the denominator is unity, i.e. $u_{0} \sim \frac{\bar{F}-E_{i}}{k T}=-\frac{0.2 \mathrm{eV}}{k T}$. Then $\sigma$ can be decreased to $10^{-19} \mathrm{~cm}^{2}$ which would now lead to $d_{\mathrm{FC}}=0.5 \mathrm{eV}$, much more reasonable. From the value of $u_{0}$ and using (8) one can now get an idea of $\varepsilon_{0}$ which is equal to $0.3 \mathrm{eV}$.

A very interesting conclusion can be drawn from these numbers. In reference [30] it was deduced from different spectroscopic techniques that the surface states of interest were located near the valence band maximum. However in the configuration coordinate diagram it is apparent that optical

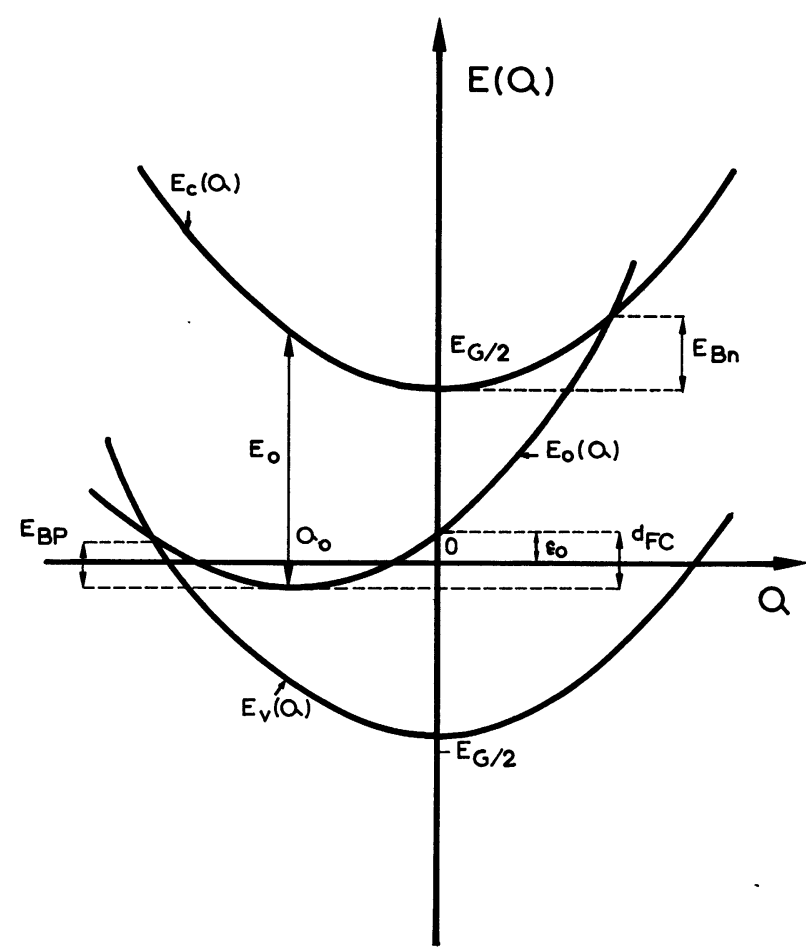

Fig. 6. - Configuration coordinate diagram (see text). $E_{0}$ is the optical threshold in a Franck-Condon transition.

transitions towards conduction band states are shifted by $d_{\mathrm{FC}}$ with respect to thermally induced transitions. This means that the apparent spectroscopic distance in energy between surface states and the conduction band is equal to $E_{\mathrm{G}} / 2-\varepsilon_{0}+2 d_{\mathrm{FC}}$ which, with the values quoted above, is equal to $1.37 \mathrm{eV}$. This exactly corresponds to $E_{\mathrm{G}}$, in good agreement with experiment.

We have thus shown that the apparent discrepancy between observed values of $S$ and theory can be easily resolved along different possible lines. In particular conventional theory of multiphonon capture can be helpful in the analysis of experimental data.

\section{Conclusion.}

We have produced evidence that pinning of the Fermi level at Schottky barriers and band offsets at semiconductor heterojunctions are related together through the self energy of dangling bonds. In a second part we have discussed in some detail conventional theory of surface recombination velocity and have shown that it can provide correct orders of magnitude. We have also made use of a semiclassical expression for the capture cross section to analyse experimental results. 


\section{Bibliographie}

[1] Heine, V., Phys. Rev. A 138 (1965) 1689.

[2] Spicer, W. E., Lindau, I., SKeath, P. and Yu, C. Y., J. Vac. Sci. Technol. 17 (1980) 1019.

[3] Stevenson, D. T. and Keyes, R. J., Physica 20 (1954) 1041.

[4] MANY, A., Goldstein Y. and Grover, N. B., in Semiconductor Surfaces (Elsevier, New York) 1971.

[5] Lannoo, M. and Bourgoin, J., Point Defects in Semiconductors I (Theoretical Aspects), Springer Series in Solid State Sciences, Vol. 22 (1981) and also Bourgoin, J. and LANNOo, M., Point Defects in Semiconductors II (Experimental Aspects), Springer Series in Solid State Sciences, Vol. 35 (1983).

[6] Johnson, N. M., Biegelsen, D. K., Moyer, M. D., Chang, S. T., PoindeXter, E. H. and Caplan, P. J., Appl. Phys. Lett. 43 (1983) 563.

[7] JACKson, W. B., Solid State Commun. 44 (1982) 477.

[8] Flores, F. and Tejedor, C., J. Physique Colloq. to be published.

[9] Sankey, O. F., Allen, R. E., Ren, S. F. and Dow, J. D., J. Vac. Sci. Technol. B 3 (1985) 1182.

[10] Tersoff, J., Phys. Rev. Lett. 52 (1984) 465, Phys. Rev. B 30 (1984) 4874, J. Vac. Sci. Technol. B 3 (1985) 1157, Phys. Rev. Lett. 25 (1986) 2755.

[11] Tejedor, C., Flores, F. and Louis, E., J. Physique Colloq. 38 (1977) C10-2163.

[12] Duggan, G., J. Vac. Sci. Technol. B 3 (1985) 1224.

[13] VAN DE WAlle, C. G. and MARTin, R. M., J. Vac. Sci. Technol. B 4 (1986) 1055.

[14] Priester, C., Allan, G. and Lannoo, M., Phys. Rev. B 33 (1986) 7386 ; HAussy, B., PrIESTER, C., Allan, G. and Lannoo, M., Phys. Rev. B, to be published.

[15] Platero, G., Sanchez-Dehesa, J., Tejedor, C. and Flores, F., Surf. Sci.,\&168 (1986) 553 ; Muñoz, A., Duran, J. C. and Flores, F., to be published.
[16] Dingle, R., in Festkörperprobleme - Advances in Solid State Physics, ed. H. R. Queisser (Vieweg, Braunschweig) 1975, Vol. 15, p. 21.

[17] Katnani, A. D. and Bauer, R. S., Phys. Rev. B 33 (1986) 1106.

[18] Wang, W. I. and Stern, F., J. Vac. Sci. Technol. B 3 (1985) 1280.

[19] Batey, J. and Wright, S. L., J. Appl. Phys. 59 (1986) 200.

[20] Sasaki, J., Chang, L. L., Ludeke, R., Chang, C. A., Sai-Halasz, G. A. and Esaki, L., Appl. Phys. Lett., 31 (1977) 211. See also CHANG, L. L. and EsAKI, L., Surf. Sci. 98 (1980) 70.

[21] Margaritondo, G., Phys. Rev. B 31 (1985) 2526.

[22] Waldrop, J. R., Kraut, E. A., KowalczyK, S. P. and Grant, R. W., Surf. Sci. 132 (1983) 513.

[23] Katnani, A. D. and Margaritondo, G., Phys. Rev. B 28 (1983) 1944.

[24] Kowalczyk, S. P., Kraut, E. A., Waldrop, J. R. and Grant, R. W., J. Vac. Sci. Technol. 21 (1982) 482.

[25] Lefebvre, I., Lannoo, M., Priester, C. and Allan, G., to be published.

[26] Pêcheur, P., Kauffer, E. and Gerl, M., Inst. Phys. Conf. Ser. 46 (1979) 174.

[27] Vogl, P., Hualmarson, H. P. and Dow, J. D., J. Phys. Chem. Solids. 44 (1983) 365.

[28] Talwar, D. N. and Ting, C. S., Phys. Rev. B 25 (1982) 2660.

[29] AsPNES, D. E., Surf. Sci. 132 (1983) 406.

[30] MoIson, J. M., VAN Rompay, M. and Bensoussan, M., Appl. Phys. Lett. 48 (1986) 1362 ; MoISON, J. M. and Bensoussan, M., to be published.

[31] Henry, C. H. and Lang, D. V., Phys. Rev. B 15 (1977) 989.

[32] LANNOO, M., Proceedings of the 13th International Conference in Semiconductors, The Metallurgical Society of AIME, Ed. L. C. Kimerling and J. M. Parsey, p. 221 (1985) 